\title{
Aesthetic Representation of Subtitle Translation in American Movies from the Perspective of Translation Aesthetics
}

\author{
Lei Liu, Mei Dong \\ Xi'an Shiyou University, Xi'an, China \\ Email:2356875375@qq.com,2548121615@qq.com
}

How to cite this paper: Liu, L. and Dong, M. (2020) Aesthetic Representation of Subtitle Translation in American Movies from the Perspective of Translation Aesthetics. Open Access Library Journal, 7: e6891. https://doi.org/10.4236/oalib.1106891

Received: October 12, 2020

Accepted: December 22, 2020

Published: December 25, 2020

Copyright $\odot 2020$ by author(s) and Open Access Library Inc.

This work is licensed under the Creative Commons Attribution International License (CC BY 4.0).

http://creativecommons.org/licenses/by/4.0/

\section{(c) (i) Open Access}

\begin{abstract}
Recently, American movies have been favored by more and more people in China. This paper takes the subtitle translation of the American film "Gifted" as an example from the perspective of translation aesthetics, and discusses the aesthetic representation of subtitle translation by adopting the $\mathrm{ABC}$ principle of translation aesthetics, in the hope of providing reference for the same type of translation and promoting the better dissemination of American films in China.
\end{abstract}

\section{Subject Areas}

Translation Aesthetics

\section{Keywords}

Translation Aesthetics, ABC Principle, Subtitle Translation, Aesthetic Representation, American Film

\section{1. 引言}

在中国翻译界, 翻译和美学的结合已有悠久的历史。在全球化的推动下, 我国观众对美国影视作品的需求越来越多, 优秀的美剧作品已成为人们了解 美国历史文化的重要途径。而翻译美学在字幕翻译中扮演着重要角色, 使得 译文即可以满足观众的审美需求，又可以促进了两国的文化交流。

\section{2. 翻译美学理论的流变及其在美国电影字幕翻译中的意义}

\section{1. 翻译美学理论的流变}

毛荣贵(2005)在《翻译美学》一书中划分了四个部分, 即主体篇、问美篇、 
朦胧篇和实践篇。他指出, 翻译审美客体有两个, 即原文 $(\mathrm{ST})$ 和译文 $(\mathrm{TL})$ 。 与之对应的有, 翻译中的三个审美主体: 译者、编辑、读者。译者是原文的 审美主体, 读者是译文的审美主体, 而编辑则既是原文又是译文的审美主体。 在这三个审美主体中, 起主导作用的是译者。[1] (p. 22)

傅仲选 1993 年发表的《实用翻译美学》一书中列举了几种常见的审美再 现手段, 比如增词法、减词法、引申法、替代法、分译法等。[2] (pp. 217-241) 他认为, “翻译还不单是语言转换。它是审美主体(译者)通过审美中介(译者 的审美意识)将审美客体(原文)转换为另一种审美客体(译文)的一种审美活 动, 因而也是思维方式的转变。也就是说, 译者应该用本民族的思维方式来 表达原文的思维内容。” [2] (p. 217)

刘宓庆(2012) [3]出版的《翻译美学导论》一书中主要探讨了翻译的审美 主体、审美客体、译学的美学洲源、审美意识、审美价值、审美表现、审美 再现、审美理想、西方美学等问题, 推动了翻译美学理论的丰富。

赵睿(2017)在《翻译的美学研究》中指出, 正是有了 “审美意识系统”, 审美客体才让审美主体投入到审美价值判断的过程中。这是 “由一个系统组 成的结构”, 是审美主体最赋能动性的结构。[4] (p. 118)他以翻译审美研究为 基本取向, 其中包括翻译的科学性研究、艺术性研究、翻译审美中的客体研 究、翻译审美中的主体研究、翻译审美中的意识系统研究以及艺术的层级研 究; 并对比了中西方的翻译美学理论, 采用 “翻译为体, 美学为用” 的原则, 丰富了现有的翻译美学理论。

\section{2. 翻译美学理论在美国电影字幕翻译中的意义}

随着全球化的发展, 越来越多美国电影进入我国市场, 一部优秀的外语 电影作品不仅可以帮助人们放松心情, 缓解压力, 高质量的汉语字幕更是英 语学习爱好者宝贵的学习素材。美国电影覆盖面广, 所以影视字幕翻译不同 于普通的文学翻译, 为了准确传达信息就需要系统的翻译理论来指导。翻译 美学可以运用美学的基本原理来指导影视字幕翻译, 不仅可以最大程度的还 原作品的内容, 还可以满足观众的审美需求, 进而为英语学习者提供一个良 好的英语学习平台。

\section{3. 翻译美学视角下《天才少女》里词语层面的审美再现}

《天才少女》是由美国福斯探照灯公司出品的一部电影。故事讲述了小 女孩玛丽有异于常人的数学天赋, 她的舅舅和外婆为此在抚养方式方面发生 了分歧的故事。电影一播出就受到观众的喜爱与支持, 同时收获了大批中国 粉丝。

刘宓庆(2012)在《翻译美学导论》中指出词是语言中能承载审美信息的一 个基本单位, 因为词是形、义、音三者的结合体, 并提出采用 “准美精” 作 为词语层面的审美标准。 “准美精” 三条审美标准, 也可简称为词的 “ABC 原则”, 即: 1) 准 (appropriateness, 简称 A)即用词准确, 既准确的表达了原 意, 又准确的适应了语境、语法和逻辑上的合理性以及用词习惯上的贴切。 
2) 美(beauty, 简称 B), 不是指可以用华丽、绚烂的等词汇, 而是指译文可以 让人身心愉悦。3) 精(compactness, 简称 C), 即精练, 用词切忌拖沓, 使用 无益的词堆砌。[3] (pp. 92-93)这一标准很适合美国电影字幕翻译的用词, 既 可以为影视字幕翻译提供理论依据, 又可以使译本展现出美感。

基于刘宓庆的 “准美精” 标准, 本文将对《天才少女》中具有审美价值 的词语进行分析, 展示翻译美学对影视字幕翻译的理论指导性作用。

\section{1）翻译美学视角下 A 原则在《天才少女》里的审美再现。}

例一. Frank: We've discussed this ad nauseam.

Mary: What's ad nauseam?

Frank: Oh, you don't know? Well, looks like someone needs school.

弗兰克: 这事讨论的没完没了。

玛丽: 没完没了是什么意思?

弗兰克: 这个词都不会? 那你需要上学了。

例一中, 玛丽到了该上学的年龄, 即使她比同龄孩子掌握了更多的知识, 他的叔叔弗兰克还是想让她去上学, 和其他普通孩子一样, 拥有一个正常的 童年。玛丽对此不能理解, 于是找了各种理由去反驳。“ “ad nauseam” 本意 为 “令人作呕地” ，在这里译为 “没完没了的” 更合适。“looks like someone needs school” 这句话中的 “someone” 本意是 “某人” , 根据剧情可知这里 的 “someone” 指的是玛丽, 所以此处译为 “你需要上学了”。

例二. come on 的三种不同译法

a) Frank: You're gonna meet kids today you can borrow money from the rest of your life. Come on. You're gonna be great.

弗兰克：你今天会结识你一辈子都可以跟他们借钱花的新同学。去吧, 你会做得很好的。

第一个 “come on” 是弗兰克鼓励玛丽去上学时说的, 表达了对玛丽即将 到来的学校生活的期许。

b) Frank: Come on, Roberta. If you start crying, I'm gonna have to pretend to start crying.

弗兰克: 别这样, 罗伯特。你要是哭, 我就只能装哭了。

第二个 “come on” 是发生在弗兰克和罗伯特的对话中, 罗伯特认为弗兰 克把玛丽送进学校是一个错误的决定, 可能会耽误一个数学天才的前途, 所 以为此感到惋惜而落泪。弗兰克理解她的用心, 此时的 “come on” 表达了一 种安慰, 所以译为 “别这样” 更合适。

c) Mary: Now can I say something I wanna say?

Bonnie: Sure.

Mary: Before they ruined it, Justin's zoo was the best art project. By far. It was awesome. Come on. A little harder.

玛丽: 我能说句真心话吗

邦妮: 好的

玛丽: 在摔坏之前, 贾斯汀做的动物园是最棒的。暂时没人超过, 做得 特别棒。来吧, 用力鼓掌。 
第三个对话发生在玛丽向全班同学解释自己为什么要为贾斯汀打抱不 平, 此时的 “come on” 表达了玛丽对贾斯汀作业的认可和赞赏, 所以希望同 学们也能认可贾斯汀为此所付出的努力, 这里译为 “来吧” 更贴切。

这三个 “come on” 发生的情景不一样, 所表达的含义也就不一样。译者 恰当的应用了 $\mathrm{A}$ 原则, 根据剧情选用最准确恰当的词语来翻译字幕, 使观众 即能够准确的接收到作者的原意, 不影响观影感受, 又可以将字幕译文作为 学习英语的材料。

\section{2）翻译美学视角下 B 原则在《天才少女》里的审美再现。}

例三. The Wind

I listen to the wind, to the wind of my soul

Where I'll end up Well, I think Only God really knows

I've sat upon the setting sun. But never, never, never, never. I never wanted water once.

No, never, never, never

歌曲: 风

我听风吹过，听我的心灵之风吹过，我想我的落脚之地只有上帝才真正 知道。我已等到夕阳西下, 但我从未, 从未, 我从未想要过水源, 从未, 从 未, 从未。

例三中, 这是弗兰克和玛丽出海时, 电影响起的一首插曲。这段译文很 有美感，比如译者将 “the wind of my soul” 译为 “心灵之风”, “where l'll end up” 没有直译为 “我最终会在哪里” ，而是意译为 “我的落脚之地”。“l've sat upon the setting sun” 这句话如果按字面意思翻译则为 “我坐在夕阳上” , 而译者译为了 “我已等到夕阳西下” 则更有诗意。这样的译文不仅符合剧情 的发展，也带给观众一种美的享受。

例四. Frank: I know. You can't hit people. That will be made very clear. I get that. But Mrs. Davis, if we separate our leaders, if we segregate them from people like you and me, you get congressmen.

弗兰克: 我知道, 打人是不对的。我会跟她明确这一点, 这我懂。但如 果把未来的领导人和普通人分开, 让他们脱离我们这样的群众, 那你就只能 得到一无是处的众议员了。

例四中，玛丽学校的校长想劝说弗兰克把玛丽送到更好的学校，因为她 认为玛丽和其他孩子不一样是一个天才，而弗兰克反驳时说了这段话。“you get congressmen” 此处译者并没有直译为 “你会得到政客” ，而是采用 B 原 则将这句话意译为 “那你就只能得到一无是处的众议员了” , 否则译文略显 突兀，很难表达出那种流畅的美感。这种美感不同于诗词歌句中的美感，而 是指译文给观众的感受。弗兰克想表达的是，即使他知道玛丽比一般孩子聪 明，他也不希望玛丽受到特别对待，希望她和同龄孩子一样接受相同的教育， 有一个正常的童年。正如即使人们知道领导和普通人不一样，但也不能让领 导脱离群众, 否则他们的存在对于普通人来说是没有意义的。译者对此进行 增词是为了更好的表达作者的原意, 虽然用词朴实, 但更能让观众理解和欣 赏。 


\section{3）翻译美学视角下 C 原则在《天才少女》里的审美再现}

例五. Robert: Frank! Frank, I know you hear me. Frank!

There's still time for you to undo this nonsense. Go get in your car and go get that child. Frank:

Robert: How can you stand there acting all calm and everything, Frank, and make light of this?

罗伯特: 弗兰克!弗兰克,我知道你能听到。弗兰克, 你现在补救还来得及, 开车去把孩子接回来。

弗兰克：我允许你可以随时用这钥匙了吗?

罗伯特：你怎么能这么淡定对此毫不在乎呢？

例五中, 当弗兰克把玛丽送到学校后, 他的朋友罗伯特对此表示不满, 她认为玛丽太聪明了, 不应该去学校, 担心玛丽去学校会变得平庸。“There's still time for you to undo this nonsense.” 这句话如果一字一句的翻译则为 “你 还有时间不去做这件没有意义的事”，这样译文就显得有些啰嗦，不如直接 译为 “你现在补救还来得及”。“Are you technically allowed to use those keys whenever you want?” 这句话译者并没有把每个单词都翻译出来, 而是选择省 译了 “technically”, 同时这句话的原意并没有改变, 反而译文显得更加简练, 观众也可以更快的获取信息。同理，还有这句话 “How can you stand there acting all calm and everything, Frank, and make light of this?” 译为 “你怎么能 这么淡定对此毫不在乎呢？”，其中译者省译了 “stand there”，“acting all calm and everything” 本意为 “表现得镇定自若” ，此处译者译为了 “淡定”， 译者在翻译时精练用词，更能直白的表达原文意思。

例六. Evelyn: I'd kill a priest for a Benadryl.

Frank: Still with the allergies?

Evelyn: Why in God's name have you got a cat? You don't even like cats.

Frank: It's not my cat. It's Mary's cat. I'm just along for the ride.

伊芙琳：我现在特想吃药。

弗兰克：还是过敏吗？

伊芙琳：你干嘛养猫？你又不喜欢猫。

弗兰克：不是我的猫，是玛丽的猫。我只是顺便一起。

例六中，这段对话发生在玛丽的外婆伊芙琳终于找到了玛丽的住处，但 她对弗兰克为自己的孙女提供的生活环境感到很不满。“kill a priest” 本意为

“杀死一个牧师”, 这里表示一种非常不满, 略带生气的语气, 所以译者选 择省译。“benadryl” 本意是指 “苯那君(药品苯海拉明的一种品牌, 伤风抗 素剂的一种)”, 这里译者直接译为 “药”。因为这句话 “I’d kill a priest for a Benadryl” 只是伊芙琳为了表达对弗兰克条件的不满, 用夸张的手法表示已经 到了要吃药的程度, 所以没有必要细究是哪一种药, 译者直接译为 “我现在 特想吃药” 更合适，也能让观众更快的理解剧情。

\section{4. 结语}

本文运用翻译美学理论, 从词语层面的 $\mathrm{ABC}$ 原则, 对《天才少女》字幕 
翻译进行了分析, 优秀的影视译本应该准确的表达作品的原意, 同时又要结 合剧情发展和中国观众的用语习惯，选用最恰当的词汇进行翻译，使译本不 仅可以给观众带来美的享受，还可以增长知识、丰富生活。

\section{Conflicts of Interest}

The authors declare no conflicts of interest regarding the publication of this paper.

\section{References}

[1] 毛荣贵. 翻译美学 [M]. 上海: 上海交通大学出版社, 2005 .

[2] 傅仲选. 实用翻译美学 $[M]$. 上海: 上海外语教育出版社, 1993.

[3] 刘宓庆. 翻译美学导论 [M]. 北京: 中国对外翻译出版有限公司, 2012: 49, 256, $261,265$.

[4] 赵睿. 翻译的美学研究 $[\mathrm{M}]$. 北京: 北京理工大学出版社, 2017.

\section{Appendix (Abstract and Keywords in Chinese) 翻译美学视角下的美国电影字幕翻译的审美再现}

摘要: 近来, 美国电影在中国受到越来越多的人喜爱。本文就翻译美学 视角下以美国电影《天才少女》的字幕翻译为例, 通过采用翻译美学的 $\mathrm{ABC}$ 原则探讨字幕译本的审美再现，以期为同类型翻译提供借鉴，促进美国电影 在中国得到更好的传播。

关键词：美国电影, 翻译美学, $\mathrm{ABC}$ 原则 